\title{
La transparencia sobre Responsabilidad Social Corporativa y su implicación con el comportamiento económico-financiero: efectos en las empresas de Economía Social
}

\author{
Javier Iturrioz del Campo ${ }^{1}$, Cristina Isabel Masa Lorenzo ${ }^{2}$ y Arturo Medina Castaño ${ }^{3}$
}

Recibido: 21 de junio de 2019 / Aceptado: 18 de julio de 2019

Resumen. Las peculiaridades de las empresas que forman parte de la Economía Social las vincula a acciones de Responsabilidad Social Corporativa (RSC). Este trabajo analiza la relación entre la publicación de datos vinculados a RSC de empresas no financieras y algunos indicadores económicofinancieros (rentabilidad económica, rentabilidad financiera, liquidez o solvencia a corto plazo, endeudamiento y tamaño). Si fuera manifiesto un efecto significativo entre ambos aspectos (RSC y performance financiero), esto supondría un impulso para las empresas de Economía Social dentro del conjunto empresarial, dada la propensión de estas hacia este tipo de acciones. Las empresas consideradas son las empresas españolas incluidas en la base de datos Bloomberg, analizando la vinculación entre su situación económico-financiera, y la publicación de información recogida en los índices de RSC, de la citada base de datos. En concreto Bloomberg proporciona cuatro índices de sostenibilidad: uno de aspectos ambientales, otro de tipo social, un tercero vinculado al gobierno corporativo y un cuarto indicador global que recoge a los tres anteriores. Se ha obtenido información de 51 empresas con datos del año 2018, estudiando las posibles diferencias entre las empresas que están incluidas o no en el IBEX. Los resultados del estudio permiten concluir que el tamaño y la liquidez son las variables que presentan mayor relación significativa con respecto a la cantidad de información publicada, y que la transparencia no afecta ni a la rentabilidad económica ni a la financiera.

Palabras clave: Responsabilidad Social Corporativa (RSC); Índices sostenibles; Economía Social; Empresas españolas; IBEX35.

Claves Econlit: G30; M14; P13; Q01.

\section{[en] Corporate Social Responsibility Transparency and its involvement with} financial-economic behaviour: effects on Social Economy companies

\begin{abstract}
The peculiarities of the companies which are part of the Social Economy link them to the actions of the Corporate Social Responsibility (CSR). This paper analyzes the relationship between the publication of data which are linked to CSR of non-financial companies and some financialeconomic indicators (economic profitability, financial profitability, liquidity or short-term solvency,
\end{abstract}

1 Universidad CEU San Pablo, España

Dirección de correo electrónico: itucam@ceu.es

2 Universidad CEU San Pablo, España

Dirección de correo electrónico: cmasalor@ceu.es

3 Universidad CEU San Pablo, España

Dirección de correo electrónico: art.medina@ceindo.ceu.es 
financial indebtedness and size). In case of having a significant effect between both aspects (CSR and financial performance), this would give a strong impetus to the Social Economy companies within the business network, given the propensity of these companies towards these kinds of actions and decisions. The companies analysed are those Spanish companies which belong to the Bloomberg database, taking into consideration the relation between their financial-economic situation and the publication of information collected from the Bloomberg database CSR indexes. To be more specific, Bloomberg provides four sustainability indexes: the first one related to environmental aspects, the second one is a social nature index, another one linked to corporate governance and the last one is a global indicator which includes the previous three ones. Data have been obtained from 51 companies in 2018, studying in detail the possible differences between the companies which are included or not on the IBEX35. The results of this research allow us to conclude that size and liquidity are the variables that have a higher significant relationship related to the amount of published information and the fact that transparency does not impact on economic profitability and financial profitability either.

Keywords: Corporate Social Responsibility (CSR); Sustainability Indexes; Social Economy; Spanish Companies; IBEX35.

Sumario. 1. Introducción. 2. RSC: concepto y evolución histórica. 3. Estudio Empírico: metodología, muestra y variables explicativas. 4. Resultados del Estudio Empírico. 5. Conclusiones. 6. Referencias bibliográficas.

Cómo citar: Iturrioz del Campo, J., Masa Lorenzo, C.I. y Medina Castaño, A. (2019) La transparencia sobre Responsabilidad Social Corporativa y su implicación con el comportamiento económico-financiero: efectos en las empresas de Economía Social. REVESCO. Revista de Estudios Cooperativos, Tercer Cuatrimestre, No 132, pp. 122-143. DOI: 10.5209/REVE.65487.

\section{Introducción}

Existe un enorme interés en el mundo académico y empresarial por todo lo relacionado con la Responsabilidad Social Corporativa (RSC), tanto por parte del sector público, nacional e internacional, como del sector privado (Charlo y Moya, 2010). Tradicionalmente la RSC ha estado muy vinculada a la Economía Social por el papel que juegan los socios de estas empresas (Comín, 2007; Lorenzo et al., 2016). Esta vinculación se hace especialmente importante en las sociedades cooperativas, en las que se produce una identificación entre los principios cooperativos y los valores que sustentan la RSC (Puentes y Velasco, 2009; Fernández y Arca, 2016; Mozas y Puentes, 2010). Independientemente del tipo de empresa, cada vez más estas interactúan con sus entornos, replanteándose productos, realizando mejoras continuas a su cadena de valor y cooperando al desarrollo local (Chivite y Enciso, 2015). Sin embargo, el hecho de que las compañías estén más preocupadas por interactuar con su entorno de manera más ética y responsable no les exime de su preocupación por la consecución de adecuados resultados económicos y financieros. Además, la obtención de información sobre RSC y su publicación implica un gasto adicional, que supone una disminución del beneficio a corto plazo, y una reducción del valor de las acciones a largo plazo (Bonin, 2003).

El cambio de valores de la sociedad, cada día más involucrada en temas sociales y medioambientales, ha provocado una reacción en las empresas, influenciadas por: la proliferación de iniciativas públicas y privadas relativas a la actuación e información en el ámbito de la RSC, por las modificaciones en las pautas de 
mercados de consumo, y por la deriva de los mercados financieros hacia inversiones socialmente responsables (Charlo et al., 2013). Los inversores incorporan, cada vez en mayor medida, criterios éticos en materia social y medioambiental a sus decisiones de inversión, lo que lleva a las empresas a implantar buenas prácticas y a informar sobre las mismas.

Por ello, en el presente trabajo se pretende analizar si existe relación entre la cantidad de información sobre el comportamiento socialmente responsable proporcionada por las empresas y su performance financiero. En el caso de ponerse de manifiesto un efecto significativo entre la transparencia en la publicación de información sobre RSC y los aspectos económico-financieros, la propensión de las empresas de economía social hacia las acciones de RSC supondría un impulso para este tipo de entidades dentro del conjunto empresarial. Para ello se analiza inicialmente de concepto y la evolución histórica de la RSC, posteriormente se recoge la metodología y las variables utilizadas en el trabajo, para finalmente analizar los resultados obtenidos y las conclusiones derivadas de los mismos.

\section{RSC: concepto y evolución histórica}

Aunque no existe una definición universalmente aceptada, la Responsabilidad Social Corporativa (RSC) (Carrol, 1999), puede entenderse como una obligación ética o moral, voluntariamente asumida por las empresas, en reconocimiento de los daños que puedan haberle sido causados a la sociedad por la actividad empresarial (Castillo, 1985). La RSC valora la capacidad de la empresa para desarrollar relaciones con el conjunto de las partes vinculadas a la misma ${ }^{4}$, en relación a aspectos sociales, financieros y medioambientales. De un tiempo a esta parte, la necesidad de considerar los intereses de todos los grupos de interés que participan en la actividad de una empresa, hace que se haya potenciado la información ofrecida por las propias empresas con respecto a estos aspectos (Archel, 2003).

En cuanto a la evolución histórica, aunque algunas empresas recogían este tipo de información de manera puntual, es en la década de los 70 del siglo pasado cuando se empieza a popularizar la inclusión de aspectos sociales dentro de los informes financieros (Steiner, 1971; Vázquez, 2015). En la década de los 90, derivado de diferentes desastres medioambientales, se presta mayor atención a este tipo de información, lo que lleva a las autoridades de países como Dinamarca o Alemania a establecer la necesidad de informar sobre las consecuencias de la actividad medioambiental de las empresas, incrementándose el número de estudios (McWilliams y Siegel, 2000). La entrada del siglo XXI popularizó la utilización del término de RSC, aunque también se utilizan otros como Desarrollo Sostenible.

La comunicación de las actividades vinculadas con la RSC por parte de las empresas puede realizarse utilizando diversos canales, aunque es el Informe de Sostenibilidad la herramienta más empleada. Este informe está asociado al término

4 El término de "Partes Interesadas" o "Grupos de interés" (stakeholders) incluye a la persona, comunidad u organización afectada por las operaciones de una entidad, o que influye en dichas operaciones. Pueden ser: internas (empleados) o externas (consumidores, proveedores, socios, comunidad local, etc.), así como los grupos de interés ausentes (generaciones futuras) a los que la actividad presente de la empresa pueda suponer alguna clase de carga o impacto. 
"triple cuenta de resultados", que consiste en integrar la información sobre los aspectos económicos, sociales y medioambientales (Elkington, 1998). Así, las empresas han elaborado informes incluyendo distintos datos de este tipo pero sin un formato definido (Adams y Evans, 2004). Para homogeneizar la información diversas organizaciones han desarrollado recomendaciones sobre la manera de incluir los aspectos de RSC y han diseñado diferentes indicadores e índices. Entre estas organizaciones se pueden destacar las siguientes:

Global Reporting Iniciative ${ }^{5}$ (GRI): Esta organización, fundada en Boston en 1997, publica una serie de estándares para los Informes de Sostenibilidad (Estándares GRI) que diferencian cuatro dimensiones: medioambiental, económica, ambiental y social. También publican un indicador de transparencia de RSC que realiza una clasificación en función de la información publicada por cada empresa.

- AccountAbility 6 : Es una firma global de consultoría y estándares que trabaja con empresas, gobiernos y organizaciones, con el objetivo de promover prácticas comerciales responsables y de mejorar su actividad a largo plazo. Publica el estándar AA1000AP cuyo objetivo es proporcionar a las organizaciones un conjunto de principios que permitan a las empresas evaluar, gestionar y comunicar su actividad en materia de sostenibilidad. Para ello ha publicado en 2018 una nueva versión (AA1000AP: 2018) que añade a los tres principios de la versión anterior (inclusividad, materialidad y capacidad de respuesta), un cuarto principio que se refiere a que las organizaciones deben medir y ser responsables la forma en la que sus acciones afectan a su entorno.

- $\quad$ Naciones Unidas $(\mathrm{ONU})^{7}$ : En 2015, la ONU aprobó la Agenda 2030, que cuenta con 17 Objetivos de Desarrollo Sostenible (ODS), en los que se consideran desde aspectos medioambientales hasta elementos vinculados a la educación. Cada objetivo tiene metas específicas afectando tanto a los gobiernos, como al sector privado.

El Observatorio de Responsabilidad Social Corporativa (ORSC) ${ }^{8}$ : Es una organización española sin ánimo de lucro que desde el año 2004 trabaja en el impulso de la correcta aplicación de la responsabilidad social corporativa. Funciona como una plataforma para la cooperación e investigación con el objetivo de potenciar la RSC en las empresas, de sensibilización y de seguimiento de las políticas públicas y sus aplicaciones prácticas.

Otro elemento a tener en cuenta con respecto a esta información es la aprobación, en 2018, de la Ley 11/20189 , que traspone la Directiva europea de

\footnotetext{
Global Reporting Initiative (https://www.globalreporting.org).

Account Ability (http://www.accountability.org).

Naciones Unidas: Objetivos de Desarrollo Sostenible (https://www.un.org/sustainabledevelopment/es/).

Observatorio de Responsabilidad Social Corporativa (https:// www.observatoriorsc.org).

Ley 11/2018, de 28 de diciembre, por la que se modifica el Código de Comercio, el texto refundido de la Ley de Sociedades de Capital aprobado por el Real Decreto Legislativo 1/2010, de 2 de julio, y la Ley 22/2015, de 20 de julio, de Auditoría de Cuentas, en materia de información no financiera y diversidad.
} 
información no financiera y diversidad ${ }^{10}$. Esta normativa ha supuesto la realización de cambios en el Código de Comercio, en la Ley de Sociedades de Capital y en la Ley de Auditoría. El resultado es la adaptación de la información en el ámbito europeo buscando, entre otros objetivos, mejorar la sostenibilidad y aumentar la confianza de los inversores, consumidores y la sociedad. Para ello, se amplía la información extrafinanciera sobre todo, de factores ambientales y sociales, así como de lucha contra la corrupción. La normativa es de obligado cumplimiento para las empresas que cumplan alguna de las siguientes condiciones:

- Superen los 500 trabajadores (o durante dos ejercicios, tengan más de 250 empleados de media).

- $\quad$ Su cifra de negocio anual sobrepase los 40 millones de euros.

- $\quad$ Las partidas del activo estén por encima de los 20 millones de euros.

- $\quad$ Entidades cuya matriz tenga sede en un país comunitario.

Estas empresas tienen que elaborar un informe especial (separado o integrado en su memoria anual) incluyendo la siguiente información:

- Medio ambiente: efectos actuales y previsibles de su impacto, uso de agua y de energía renovable y/o no renovable, e información sobre emisiones contaminantes.

- Cuestiones sociales: medidas para garantizar la igualdad de género, sobre las condiciones de trabajo, el diálogo social, el respeto de los derechos sindicales, la salud y seguridad en el trabajo y las relaciones con las comunidades locales.

- Lucha contra la corrupción y el soborno: información sobre los procedimientos de control interno para prevenir este tipo de conductas.

- Diversidad: medidas aplicadas en relación con: formación, experiencia profesional, edad, discapacidad y género, así como objetivos y resultados para que en el consejo de administración exista un número equilibrado de mujeres y hombres.

Los diferentes estudios realizados sobre la RSC diferencian entre dos teorías relacionadas con los motivos que llevan a las empresas a publicar información sobre aspectos de responsabilidad social: los basados en la Teoría de los Grupos de Interés (stakeholders) y los basados en la Teoría de la Legitimidad:

Teoría de los Grupos de Interés. Dentro de esta teoría se pueden diferenciar entre los trabajos que se centran en la relación bilateral entre los propietarios y los directivos de las empresas (Teoría de la Agencia) (Jensen y Meckling, 1976), y los que analizan la problemática ampliando la relación con otros grupos de interés (Kurucz et al., 2008). Así, la publicación de información vinculada a la responsabilidad social tiene como objetivo ofrecer información a diferentes colectivos (como trabajadores o clientes) y no solo a los propietarios o acreedores interesados en el comportamiento de la sociedad (Patten, 1992; Campbell, 2004).

10 Directiva 2014/95/UE del Parlamento Europeo y del Consejo, de 22 de octubre de 2014, por la que se modifica la Directiva 2013/34/UE en lo que respecta a la divulgación de información no financiera e información sobre diversidad. 
- Teoría de la Legitimidad. Se trata de analizar la publicación de información por parte de las empresas con el objetivo de legitimar su actividad ante la sociedad (Patten, 1992; Campbell et al., 2003; Kim, 2019; Johnson-Young y Magee, 2019). Uno de los aspectos que determinan la mayor propensión a difundir este tipo de datos es el efecto de la actividad de la empresa sobre los efectos medioambientales (Cho y Pattem, 2007).

En relación a la vinculación de la RSC y los aspectos económico-financieros, la Teoría de los Grupos de Interés establecía inicialmente que las acciones de gobierno corporativo debían orientarse a la obtención de beneficios económicos, ya que en caso contrario supondrían un incremento de costes para la empresa (Anderson, 1989). Posteriormente se planteó que estas acciones pueden proporcionar ventajas competitivas para la empresa y, en consecuencia, contribuir a su resultado económico-financiero. La teoría de la legitimidad pone el acento en aspectos sociales más que económicos. Son numerosos los estudios científicos que relacionan la RSC y el performance financiero de la empresa. Los mismos se inician en los años setenta con unos resultados no homogéneos (Friedman, 1970; Moskowitz, 1972), y desde entonces se han realizado distintas investigaciones con resultados no concluyentes y, en algunos casos contradictorios: correlaciones negativas (Nelling y Webb, 2009), correlaciones nulas (Aupperle et al., 1985; McWilliams y Siegel, 2001) y correlaciones positivas (Waddock y Graves, 1997; Mahon y Mcgovan, 1998; Orlitzky et al., 2003; Allouche y Laroche, 2005; Barnett, 2007). También se han realizado trabajos centrados en el efecto de la publicación de información socialmente responsable sobre el valor de las empresas o su rentabilidad. Así, se analiza la relación con la rentabilidad económica (Ullman, 1985), o con diversos indicadores (Bebchuk y Weisbach, 2010; De Viliers et al, 2011; Harjoto et al., 2017; Ahmadi et al., 2018; Medina e Iturrioz, 2018). Por último, mencionar que hay un considerable número de trabajos en torno a la RSC de las empresas españolas cotizadas (Vázquez y Cordero, 2007; Nieto et al., 2012; De la Cuesta y Valor, 2013; Pérez et al., 2013; Testera y Cabeza, 2013).

El efecto del tipo de empresa sobre la información relativa a la sostenibilidad es analizado para diferentes organizaciones como las empresas familiares (López et al., 2019) o las empresas cotizadas (Domench, 2003; Dey, 2007; Chang et al., 2018). En lo que respecta a las empresas de Economía Social, los estudios se han centrado en las sociedades cooperativas (García et al., 2011; Socias y Horrach, 2013; Carrasco, 2007). La conclusión generalizada de estos trabajos es que las sociedades cooperativas presentan una mejor situación estratégica ante la RSC (Collado, 2006), destacando la tradición de este tipo de empresas a combinar la viabilidad económica con la responsabilidad social, gracias a la participación de los socios en los distintos flujos empresariales (Castro, 2006). Por tanto, si se establece una relación significativa entre los aspectos económico-financieros y la RSC, la vinculación entre las empresas de economía social y la RSC puede suponer un elemento positivo para este tipo de entidades.

Por otra parte, son muchos los estudios que han analizado este aspecto desde perspectivas diferentes. Por lo que respecta a la cantidad de información publicada, algunos trabajos se han centrado en la posible relación entre la misma y la concentración del capital llevando a resultados no concluyentes (Hoopes y Miller, 
2006; Ndemanga y Koffi, 2009; Barnea y Rubin, 2010). En cuanto a los factores que pueden estar relacionados con la cantidad de información publicada, determinados estudios llevan a la conclusión de que la normativa legal y los usos y costumbres son los factores más vinculados (Gray et al., 1997; Cuevas et al., 2013).

\section{Estudio Empírico: metodología, muestra y variables explicativas}

\subsection{Metodología del Estudio y Muestra}

El objetivo de este estudio consiste en un análisis de la posible relación entre determinados indicadores de RSC (en sus diferentes ámbitos medioambiental, social y laboral, y de gobierno corporativo), con indicadores financieros derivados de los estados contables de las empresas.

Para ello, se ha analizado la relación mediante el estudio de los coeficientes de correlación entre los diferentes índices de RSC publicados por Bloomberg ${ }^{11}$ y las variables de performance financiero. Previamente, se ha realizado un análisis descriptivo de las variables utilizadas: por un lado, los indicadores de RSC proporcionados por la Plataforma Bloomberg y, por otro, los ratios de rentabilidad económica, rentabilidad financiera, liquidez, endeudamiento y el tamaño de la empresa.

El citado proceso se aplica a la muestra completa de las empresas españolas recogidas en la base de datos Bloomberg y a las dos submuestras que la forman: por un lado, a las que a su vez están incluidas en el IBEX $35^{12} \mathrm{y}$, por otro, a las que no se encuentran recogidas en el mismo. Está división se realiza con el objetivo de que analizar las diferencias entre ambos grupos (Arimany et al., 2013), teniendo en cuenta que las empresas de economía social pueden presentar una mayor afinidad con el segundo subgrupo.

La muestra total de empresas en el momento de la consulta (enero de 2019) ascendía a 161 empresas, de las que se han excluido las 14 entidades financieras (7 de ellas incluidas en el IBEX), para evitar distorsiones debido a sus peculiaridades contables y financieras (Madorran y García, 2016). De las 147 restantes, la Plataforma Bloomberg, recoge información en los índices de sostenibilidad de 51 empresas, que componen la muestra utilizada, lo que supone aproximadamente un 35 por ciento del total. De estas 51 entidades, 29 (un 56,8\%) están incluidas en el IBEX 35), y 22 (un 43,2\%) no cotizan en el citado índice.

Al igual que en otros trabajos previos, para el análisis estadístico de los datos se han utilizado coeficientes de correlaciones bivariadas, obtenidos con el programa Informático SPSS. La Tabla 1 detalla la ficha técnica de la investigación:

11 La empresa Bloomberg permite a través de sus terminales informáticos acceder, consultar y analizar información financiera de los mercados en tiempo real, y realizar operaciones de compra venta de activos en su plataforma electrónica. Además, la compañía cuenta con un canal de televisión, una Página Web y una aplicación.

12 El IBEX 35 está compuesto por los 35 valores más líquidos cotizados en el Sistema de Interconexión Bursátil Electrónico (SIBE) de las cuatro Bolsas Españolas (Madrid, Barcelona, Bilbao y Valencia), usado como referente nacional e internacional. 
Tabla. 1. Ficha Técnica de la Investigación.

\begin{tabular}{|l|l|}
\hline Universo (Población) & Empresas cotizadas \\
\hline Tamaño Muestral & $\begin{array}{l}51 \text { empresas con índices de RSC recogidas en } \\
\text { Bloomberg: } \\
\text { 29 empresas cotizadas en el IBEX 35 } \\
\text { 22 empresas no pertenecientes al IBEX 35 }\end{array}$ \\
\hline $\begin{array}{l}\text { Técnica de obtención de la } \\
\text { información }\end{array}$ & Plataforma Bloomberg \\
\hline Horizonte Temporal & 2018 \\
\hline Técnica de Análisis & Coeficientes de Correlaciones (bivariadas) \\
\hline Software Informático & IBM SPSS Statistics (versión 25.0) \\
\hline
\end{tabular}

Fuente: Elaboración propia.

\subsection{Variables Explicativas del Modelo}

Uno de los aspectos de mayor relevancia en cualquier estudio es la selección de variables independientes elegidas. A continuación, se definen cada uno de los indicadores, comenzando por los índices de sostenibilidad y RSC proporcionados por la Plataforma Bloomberg y, posteriormente, se detallan determinados ratios relacionados con el performance financiero de la empresa, elegidos tras la revisión bibliográfica realizada.

Los índices de sostenibilidad son indicadores que recogen información vinculada con el desarrollo sostenible y la responsabilidad corporativa. Estos índices se han convertido en una referencia para los inversores que, entre sus criterios de decisión, incluyen las empresas que incorporan políticas vinculadas con estos aspectos. Sin embargo, la metodología de las calificaciones varía mucho de un proveedor a otro (Escrig et al., 2010; Halbritter y Dorfleitner, 2015). Entre las diferentes alternativas (Sun et al., 2011) destacan ${ }^{13}$ :

El Indicador de calidad de información de la RSC de las empresas del $I B E X$, proporcionado por el Observatorio de la Responsabilidad Social Corporativa (ORSC): Analiza como contenidos de la RSC el medioambiente, los derechos humanos y derechos laborales, el consumo (responsabilidad sobre el producto y derechos del consumidor), las relaciones con la comunidad, la responsabilidad fiscal y gobernanza empresarial y la corrupción. Para ello, tiene en cuenta las siguientes referencias, principios, declaraciones y normas de determinados organismos: Global Reporting Initiative (GRI), Código Unificado del Buen Gobierno de la Comisión Nacional del Mercado de Valores (CNMV), Normas de Naciones Unidas sobre derechos humanos para empresas multinacionales, Requisitos y principios AA1000 del Institute of Social and Ethical Accountability, y el Modelo de New Economics Foundation (NEF).

13 Otros índices son: KLD's Domini 400 Social Index; Ethibel; Social Calvert; y ARESE Sustainable Performance. 
- El Dow Jones Sustantiability Index (DJSI): Desde su lanzamiento en 1998, es uno de los principales referentes en cuanto a "inversión sostenible". El índice general incluye, de entre las 2.500 compañías más grandes del mundo, a las que cumplen de forma habitual con una serie de requisitos económicos, medioambientales y sociales. También elabora varios índices territoriales. Se revisa anualmente.

El FTSE4 Good: Incluye a las empresas que trabajan por la sostenibilidad medioambiental, cuidan las relaciones positivas con los grupos de interés y defienden los derechos humanos. Este índice excluye a las empresas relacionadas con una serie de aspectos como el tabaco o el armamento. Se revisa dos veces al año.

Bloomberg Social and Corporate Governance Data Service (ESG): La plataforma de información Bloomberg recopila, revisa y actualiza los datos ambientales, sociales y de gobierno corporativo de más de 11.300 empresas pertenecientes a más de 80 países (Park y Ravenel, 2013). Para ello, se basa en la información publicada por las empresas, a las que posteriormente envía los datos de ESG que pretende publicar, otorgándole a las entidades la oportunidad de comprobarlos y corregirlos.

En este trabajo se toma como referencia la información elaborada por Bloomberg que ofrece los ratios clave y los indicadores de rendimiento más importantes para que los usuarios puedan comparar y analizar la actividad de las empresas en lo referente al desarrollo sostenible y al rendimiento de las empresas. Así, tiene en cuenta más de 700 categorías relacionadas con la sostenibilidad que se resumen en cuatro índices: uno de aspectos ambientales, otro de tipo social y laboral, un tercero vinculado al gobierno corporativo y un cuarto indicador global que recoge a los tres anteriores. Los principales parámetros recogidos en estos índices se encuentran resumidos en la Tabla 2.

Tabla. 2. Índices de ESG elaborados por Bloomberg.

\begin{tabular}{|c|c|c|}
\hline \multicolumn{3}{|c|}{$\begin{array}{l}\text { ÍNDICE GLOBAL } \\
\text { (ESG DISCLOSURE SCORE) }\end{array}$} \\
\hline $\begin{array}{l}\text { ÍNDICE AMBIENTAL } \\
\text { (ENVIRON_DISCLOSURE } \\
\text { _SCORE) }\end{array}$ & $\begin{array}{l}\text { ÍNDICE SOCIAL } \\
\text { (SOCIAL_DISCLOSURE_ } \\
\text { SCORE) }\end{array}$ & $\begin{array}{l}\text { ÍNDICE GOBIERNO } \\
\text { CORPORATIVO } \\
\text { (GOVNCE_DISCLOSURE } \\
\text { SCORE) }\end{array}$ \\
\hline $\begin{array}{l}\text { Emisiones de carbono. } \\
\text { Efectos del cambio } \\
\text { climático. } \\
\text { Contaminación } \\
\text { Eliminación de residuos. } \\
\text { Agotamiento de los recursos. } \\
\text { Energía renovable. }\end{array}$ & $\begin{array}{l}\text { Derechos humanos. } \\
\text { Cadena de suministro. } \\
\text { Discriminación. } \\
\text { Contribuciones políticas. } \\
\text { Defensa de la diversidad. }\end{array}$ & $\begin{array}{l}\text { Derechos de voto. } \\
\text { Relaciones con los grupos } \\
\text { de interés. } \\
\text { Compensación ejecutiva. } \\
\text { Composición de las juntas. } \\
\text { Independencia de los } \\
\text { directores. } \\
\text { Derechos de los accionistas }\end{array}$ \\
\hline
\end{tabular}

Fuente: Elaboración propia. 
Los índices recogen una puntuación recogida en el intervalo: 0,1 (cuando ofrecen una información de ESG mínima) y 100 (para las empresas que hacen públicos todos los datos recopilados por Bloomberg). Cada dato tiene una ponderación distinta según el sector. Por tanto, los índices miden la cantidad de información relativa a la ESG que las empresas declaran públicamente, pero no entra en la valoración del cumplimiento de los distintos aspectos.

A continuación, se definen las variables dependientes cuantitativas económicofinancieras que se utilizan en el análisis, resultado de la recopilación realizada sobre los principales estudios que versan sobre el objeto de estudio (rentabilidad económica, rentabilidad financiera, liquidez, endeudamiento y tamaño), definidas como ratios ${ }^{14}$, así como su forma de cálculo:

Rentabilidad Económica (ROA). Mide, en porcentaje, la renta generada por la empresa en el desarrollo de su actividad a través de los medios económicos o inversiones con los que cuenta. Indica la capacidad que tiene la empresa para generar recursos disponibles, por lo que interesa que sea positiva (solo es negativa cuando hay pérdidas de explotación), y lo más elevada posible.

$$
\text { ROA }=\text { (Resultado Explotación (BAII)/Activo Total }) \times 100
$$

Rentabilidad Financiera (ROE). En este caso se toma como renta generada por la empresa el beneficio final, una vez deducidos todos los gastos, incluido el impuesto (Beneficio Después de Impuestos, BDI). Hace referencia a la parte del resultado que, salvo por el cumplimiento de obligaciones legales, la empresa puede destinar en caso de ser positivo a autofinanciación (reservas), o a remunerar a los propietarios (dividendos). Por ello, al ponerla en relación con los recursos propios, se dice que es una rentabilidad del accionista, en el sentido que indica la relación con el beneficio final y los recursos propios. Por tanto, interesa que este indicador resulte positivo y lo más alto posible.

$\mathrm{ROE}=($ Resultado del Ejercicio $(\mathrm{BDI}) /$ Fondos propios $) \times 100$

- $\quad$ Liquidez. Este ratio, también denominado índice de solvencia en el corto plazo, mide la capacidad de la empresa para atender a sus deudas a corto plazo, o dicho de otra manera la garantía que ofrece a sus acreedores en el corto plazo. Un valor próximo a la unidad no garantiza que la empresa pueda hacer frente a sus compromisos más inminentes, mostrando una situación de riesgo extremo. Interesa que sea lo más alto posible, y cuando su resultado es superior a la unidad, quiere decir que la entidad es capaz de atender con sus inversiones corrientes sus deudas de corto plazo, minimizándose el riesgo que supondría que parte de las inversiones de corto no se convirtieran en liquidez. Por el contrario, en el caso de ser inferior a uno, indica posible situación de preconcurso y, económicamente, indica suspensión de pagos,

14 Los ratios son los principales indicadores utilizados en el análisis financiero de la empresa (Sanz, 2002). Se definen como cocientes entre dos magnitudes contables con el objetivo de obtener como resultado información que pueda ser comparable, bien en distintos momentos del tiempo para una misma empresa, o bien entre una serie de empresas a lo largo de un período o en un momento puntual. Por tanto, los ratios proporcionan la adimensionalidad necesaria para evitar los efectos de las unidades de medida, presentes cuando se trabaja con valores absolutos, permitiendo así la comparabilidad en un determinado momento del tiempo de distintas empresas independientemente, tal y como se hace en esta investigación. 
pues la empresa no va a poder hacer frente con sus inversiones de corto a las deudas que vencen en este mismo plazo. Esta situación supone además un fondo de maniobra o capital circulante negativo, manifestando una inestabilidad financiera clara.

LIQUIDEZ=Activo Corriente/Pasivo Corriente

- Endeudamiento. Este indicador refleja la medida del riesgo financiero de la empresa, mostrando la proporción de la estructura financiera elegida por la misma, que está formada por financiación ajena o de terceros. Cuanto más elevado sea este indicador más apalancada o endeudada estará la empresa, dado que tiene que hacer frente a más recursos financieros y esto implica, en principio, una reducción en la calidad crediticia, y un incremento de los gastos financieros, suponiendo que la financiación ajena sea remunerada.

ENDEUDAMIENTO=Pasivo / (Patrimonio Neto + Pasivo)

Tamaño: El criterio utilizado en este análisis para medir el tamaño empresarial de las sociedades objeto de estudio ha sido el volumen de activo empresarial o balance general en miles de euros. Como en otros estudios (Jacobs et al., 2012; Charlo et al., 2013), se ha tomado el logaritmo neperiano de estas variables para que la distribución de los datos presentara normalidad, y evitar una posible asimetría de los mismos, así como la influencia de los valores atípicos (outliers).

A continuación, se presenta el resumen descriptivo de las variables económicofinancieras utilizadas como descriptivos del performance financiero de las empresas analizadas:

Tabla. 3. Estadísticos descriptivos de las Variables de Performance Financiero.

\begin{tabular}{|c|c|c|c|c|c|}
\hline VARIABLES & EMPRESAS & Media & Desv. Típica & Mínimo & Máximo \\
\hline \multirow{3}{*}{ RSC GLOBAL } & TOTAL & 45,17 & 12,51 & 16,94 & 72,83 \\
\hline & En IBEX & 49,49 & 10,57 & 28,93 & 72,83 \\
\hline & No IBEX & 39,76 & 13,05 & 16,94 & 59,92 \\
\hline \multirow{3}{*}{ RSC MEDIOAMB } & TOTAL & 38,22 & 16,42 & 0,00 & 71,72 \\
\hline & En IBEX & 43,79 & 14,01 & 12,40 & 71,72 \\
\hline & No IBEX & 31,36 & 17,33 & 0,00 & 58,91 \\
\hline \multirow{3}{*}{ RSC SOCIAL } & TOTAL & 50,24 & 14,59 & 17,54 & 80,70 \\
\hline & En IBEX & 53,38 & 14,47 & 31,58 & 78,13 \\
\hline & No IBEX & 46,17 & 13,69 & 17,54 & 68,42 \\
\hline \multirow{3}{*}{ RSC GOBIERNO } & TOTAL & 55,99 & 6,89 & 37,50 & 71,43 \\
\hline & En IBEX & 58,14 & 6,15 & 48,21 & 71,43 \\
\hline & No IBEX & 52,60 & 6,72 & 37,50 & 66,07 \\
\hline \multirow{2}{*}{ ROA } & TOTAL & 7,10 & 7,05 & $-14,24$ & 26,50 \\
\hline & En IBEX & 7,21 & 6,03 & $-5,33$ & 21,32 \\
\hline
\end{tabular}




\begin{tabular}{|l|l|l|l|l|l|}
\hline & No IBEX & 6,96 & 8,37 & $-14,24$ & 26,50 \\
\hline \multirow{4}{*}{ ROE } & TOTAL & 18,50 & 33,25 & $-26,81$ & 212,32 \\
\cline { 2 - 6 } & En IBEX & 20,61 & 38,19 & $-19,34$ & 212,32 \\
\cline { 2 - 6 } & No IBEX & 15,72 & 25,95 & $-26,81$ & 115,57 \\
\hline \multirow{4}{*}{ EIQUIDEZ } & TOTAL & 1,56 & 0,90 & 0,49 & 5,21 \\
\cline { 2 - 6 } & En IBEX & 1,54 & 1,04 & 0,49 & 5,21 \\
\cline { 2 - 6 } & No IBEX & 1,60 & 0,69 & 0,55 & 3,48 \\
\hline \multirow{4}{*}{ TAMANEUDAM. } & TOTAL & 0,63 & 0,19 & 0,23 & 1,05 \\
\cline { 2 - 6 } & En IBEX & 0,64 & 0,18 & 0,24 & 1,05 \\
\cline { 2 - 6 } & No IBEX & 0,61 & 0,21 & 0,23 & 0,98 \\
\hline & TOTAL & 8,55 & 1,59 & 4,93 & 11,64 \\
\cline { 2 - 6 } & En IBEX & 9,33 & 1,30 & 6,87 & 11,64 \\
\cline { 2 - 6 } & No IBEX & 7,51 & 1,34 & 4,93 & 10,03 \\
\hline
\end{tabular}

Fuente: Elaboración propia a partir de los datos obtenidos con IBM SPSS Statistics (versión 25.0).

Los datos descriptivos muestran que las empresas que forman parte del IBEX publican (en media) más información vinculada a la RSC en todos los índices y, como es lógico, también tienen un mayor tamaño. En cuanto a los diferentes indicadores económico-financieros, estas empresas presentan una media superior en todos excepto en la liquidez, en la que la media de las que no pertenecen al selectivo es superior.

\section{Resultados del Estudio Empírico}

El análisis realizado para determinar si existen correlaciones claras en cuanto a indicadores financieros favorables y a sus índices de RSC se presenta distinguiendo el índice de sostenibilidad utilizado. A su vez, para cada uno de los índices se detallan los datos aplicados al total de la muestra, diferenciando posteriormente posibles discrepancias entre los resultados obtenidos por estas empresas en función de las submuestras que se refieren a la pertenencia o no al IBEX 35.

\subsection{Relación entre Índice RSC Global y Resultados Económico-Financieros}

Tabla. 4. Correlaciones entre RSC GLOBAL y Performance Financiero.

\begin{tabular}{|l|l|l|l|l|l|l|l|}
\hline $\begin{array}{l}\text { CORRELACIÓN } \\
\text { DE PEARSON }\end{array}$ & $\begin{array}{l}\text { RSC } \\
\text { GLOB }\end{array}$ & ROA & ROE & LIQ & END & TAM \\
\hline RSC \\
GLOB & TOTAL & 1 & & & & & \\
\cline { 2 - 8 } & En IBEX & 1 & & & & & \\
\hline
\end{tabular}




\begin{tabular}{|c|c|c|c|c|c|c|c|}
\hline & No IBEX & 1 & & & & & \\
\hline \multirow{3}{*}{ ROA } & TOTAL & $-0,028$ & 1 & & & & \\
\hline & En IBEX & $-0,278$ & 1 & & & & \\
\hline & No IBEX & 0,135 & 1 & & & & \\
\hline \multirow{3}{*}{ ROE } & TOTAL & $-0,680$ & 0,235 & 1 & & & \\
\hline & En IBEX & $-0,302$ & $-0,113$ & 1 & & & \\
\hline & No IBEX & 0,184 & $0,762 * *$ & 1 & & & \\
\hline \multirow{3}{*}{ LIQ } & TOTAL & $-0,339^{*}$ & 0,089 & $-0,152$ & 1 & & \\
\hline & En IBEX & $-0,299$ & 0,152 & $-0,182$ & 1 & & \\
\hline & No IBEX & $-0,500^{*}$ & 0,012 & $-0,059$ & 1 & & \\
\hline \multirow{3}{*}{ END } & TOTAL & 0,256 & $-0,304 *$ & $0,369 * *$ & $-0,511^{* *}$ & 1 & \\
\hline & En IBEX & 0,059 & $-0,637 * *$ & $0,390^{*}$ & $-0,500 * *$ & 1 & \\
\hline & No IBEX & $0,432 *$ & $-0,030$ & 0,356 & $-0,576^{* *}$ & 1 & \\
\hline \multirow{3}{*}{ TAM } & TOTAL & $0,630^{* *}$ & $-0,212$ & $-0,087$ & $-0,366^{* *}$ & $0,388^{* *}$ & 1 \\
\hline & En IBEX & $0,502 * *$ & $-0,293$ & $-0,238$ & $-0,273$ & 0,161 & 1 \\
\hline & No IBEX & $0,596 * *$ & $-0,255$ & $-0,012$ & $-0,746^{* *}$ & $0,733^{* *}$ & 1 \\
\hline
\end{tabular}

*. La correlación es significante al nivel 0,05 (bilateral).

**. La correlación es significativa al nivel 0,01 (bilateral).

Fuente: Elaboración propia a partir de los datos obtenidos con IBM SPSS Statistics (versión 25.0).

Respecto al índice de RSC Global que recoge todo tipo de información sobre RSC (como variable dependiente) y las rentabilidades económica y financiera (como variables independientes) se detecta que no hay una relación lineal ni en la muestra total ni en las submuestras que recogen las empresas pertenecientes al IBEX y las no pertenecientes a este índice bursátil. Por tanto, la rentabilidad obtenida no presenta una relación lineal con la cantidad de información recogida en el índice global de RSC.

En cuanto a la liquidez, se detecta una relación lineal negativa significativa al nivel del $5 \%$, en la muestra total y solo dentro de la submuestra de las pertenecientes al IBEX. Es decir, en los grupos de empresas mencionados, cuánta más información sobre RSC publican, menor es su liquidez.

En el endeudamiento, solo aparece como significativo al 5\% la relación con las empresas que no se encuentran incluidas en el IBEX, sin que la relación sea significativa para el otro subgrupo ni para la muestra total.

Por último, el tamaño es la variable independiente que presenta una relación más significativa (al 1\%), afectando con valores similares a los dos subgrupos y con mayor efecto en la muestra total. Este efecto puede deberse a que las empresas de mayor tamaño son más susceptibles de sufrir daños en su reputación que las 
empresas de menor tamaño, por lo que tienden a publicar más información sobre los aspectos de RSC que en empresas pequeñas (Charlo et al., 2013).

\subsection{Relación entre Índice RSC Medioambiental y Resultados Económico- Financieros}

Tabla. 5. Correlaciones entre RSC Medioambiental y Performance Financiero.

\begin{tabular}{|c|c|c|c|c|c|c|c|}
\hline \multicolumn{2}{|c|}{$\begin{array}{l}\text { CORRELACIÓN } \\
\text { PEARSON }\end{array}$} & \begin{tabular}{|l} 
RSC \\
MED \\
\end{tabular} & ROA & ROE & LIQ & END & TAM \\
\hline \multirow{3}{*}{$\begin{array}{l}\text { RSC } \\
\text { MED }\end{array}$} & TOTAL & 1 & & & & & \\
\hline & En IBEX & 1 & & & & & \\
\hline & No IBEX & 1 & & & & & \\
\hline \multirow{3}{*}{ ROA } & TOTAL & $-0,080$ & 1 & & & & \\
\hline & En IBEX & $-0,298$ & 1 & & & & \\
\hline & No IBEX & 0,046 & 1 & & & & \\
\hline \multirow{3}{*}{ ROE } & TOTAL & $-0,072$ & 0,235 & 1 & & & \\
\hline & En IBEX & $-0,261$ & $-0,113$ & 1 & & & \\
\hline & No IBEX & 0,104 & $0,762 * *$ & 1 & & & \\
\hline \multirow{3}{*}{ LIQ } & TOTAL & $-0,355^{*}$ & 0,089 & $-0,152$ & 1 & & \\
\hline & En IBEX & $-0,314$ & 0,152 & $-0,182$ & 1 & & \\
\hline & No IBEX & $-0,520 *$ & 0,012 & $-0,059$ & 1 & & \\
\hline \multirow{3}{*}{ END } & TOTAL & 0,271 & $-0,304^{*}$ & $0,369 * *$ & $-0,511^{* *}$ & 1 & \\
\hline & En IBEX & 0,115 & $-0,637 * *$ & $0,390^{*}$ & $-0,500 * *$ & 1 & \\
\hline & No IBEX & 0,405 & $-0,030$ & 0,356 & $-0,576 * *$ & 1 & \\
\hline \multirow{3}{*}{ TAM } & TOTAL & $0,636 * *$ & $-0,212$ & $-0,087$ & $-0,366^{* *}$ & $0,388^{* *}$ & 1 \\
\hline & En IBEX & $0,523^{* *}$ & $-0,293$ & $-0,238$ & $-0,273$ & 0,161 & 1 \\
\hline & No IBEX & $0,601 * *$ & $-0,255$ & $-0,012$ & $-0,746^{* *}$ & $0,733 * *$ & 1 \\
\hline
\end{tabular}

*. La correlación es significante al nivel 0,05 (bilateral).

**. La correlación es significativa al nivel 0,01 (bilateral).

Fuente: Elaboración propia a partir de los datos obtenidos con IBM SPSS Statistics (versión 25.0).

Si se toma como referencia el índice de RSC que recoge solo aspectos Medioambientales (como variable dependiente), se observa que coincide con el caso anterior en tres aspectos. Por un lado, no aparece una relación significativa ni con la rentabilidad económica ni con la financiera. Por otro, el tamaño de la empresa es la única variable que presenta una relación significativa al 1\% para la 
muestra total y las dos submuestras. La tercera característica en común es la significatividad negativa al $5 \%$ con respecto a la liquidez que se produce para la muestra total y para las empresas que no pertenecen al IBEX.

Sin embargo, en el caso de la publicación de información medioambiental, no se muestra relación significativa con respecto al endeudamiento.

\subsection{Relación entre Índice RSC Social y Resultados Económico-Financieros}

Tabla. 6. Correlaciones entre RSC Social y Performance Financiero.

\begin{tabular}{|c|c|c|c|c|c|c|c|}
\hline \multicolumn{2}{|c|}{$\begin{array}{l}\text { CORRELACIÓN } \\
\text { PEARSON }\end{array}$} & \multirow{2}{*}{\begin{tabular}{|l} 
RSC \\
SOCIAL \\
1 \\
\end{tabular}} & \multirow[t]{2}{*}{ ROA } & \multirow[t]{2}{*}{ ROE } & \multirow[t]{2}{*}{ LIQ } & \multirow[t]{2}{*}{ END } & \multirow[t]{2}{*}{ TAM } \\
\hline \multirow{3}{*}{$\begin{array}{l}\text { RSC } \\
\text { SOCIAL }\end{array}$} & TOTAL & & & & & & \\
\hline & En IBEX & 1 & & & & & \\
\hline & No IBEX & 1 & & & & & \\
\hline \multirow{3}{*}{ ROA } & TOTAL & 0,045 & 1 & & & & \\
\hline & En IBEX & $-0,219$ & 1 & & & & \\
\hline & No IBEX & 0,316 & 1 & & & & \\
\hline \multirow{3}{*}{ ROE } & TOTAL & $-0,016$ & 0,235 & 1 & & & \\
\hline & En IBEX & $-0,212$ & $-0,113$ & 1 & & & \\
\hline & No IBEX & 0,334 & $0,762 * *$ & 1 & & & \\
\hline \multirow{3}{*}{ LIQ } & TOTAL & $-0,162$ & 0,089 & $-0,152$ & 1 & & \\
\hline & En IBEX & $-0,060$ & 0,152 & $-0,182$ & 1 & & \\
\hline & No IBEX & $-0,387$ & 0,012 & $-0,059$ & 1 & & \\
\hline \multirow{3}{*}{ END } & TOTAL & 0,169 & $-0,304 *$ & $0,369 * *$ & $-0,511^{* * *}$ & 1 & \\
\hline & En IBEX & $-0,020$ & $-0,637 * *$ & $0,390^{*}$ & $-0,500 * *$ & 1 & \\
\hline & No IBEX & 0,383 & $-0,030$ & 0,356 & $-0,576^{* *}$ & 1 & \\
\hline \multirow{3}{*}{ TAM } & TOTAL & $0,415^{* *}$ & $-0,212$ & $-0,087$ & $-0,366^{* *}$ & $0,388^{* *}$ & 1 \\
\hline & En IBEX & 0,225 & $-0,293$ & $-0,238$ & $-0,273$ & 0,161 & 1 \\
\hline & No IBEX & $0,513 *$ & $-0,255$ & $-0,012$ & $-0,746 * *$ & $0,733 * *$ & 1 \\
\hline
\end{tabular}

*. La correlación es significante al nivel 0,05 (bilateral).

**. La correlación es significativa al nivel 0,01 (bilateral).

Fuente: Elaboración propia a partir de los datos obtenidos con IBM SPSS Statistics (versión 25.0).

Si se analiza la información de RSC Social publicada, tan solo aparece relación significativa con respecto al tamaño. Además, esta relación es significativa al $1 \%$ 
para la muestra total y solo al $5 \%$ en la submuestra de las no pertenecientes al IBEX. Este resultado pone de manifiesto que cuanto mayor es el tamaño de las empresas más información de tipo social publican. Sin embargo, en el subgrupo de las cotizadas en el IBEX, que son las que cuentan con una dimensión mayor, el tamaño no es un elemento determinante, mientras que en las que se encuentran fuera del citado índice, y que por tanto tienen menor tamaño, sí hay una relación con la información social publicada.

\subsection{Relación entre Índice RSC Gobierno Corporativo y Resultados Económico-Financieros}

Tabla. 7. Correlaciones entre RSC Gobierno Corporativo y Performance Financiero.

\begin{tabular}{|c|c|c|c|c|c|c|c|}
\hline \multicolumn{2}{|c|}{$\begin{array}{l}\text { CORRELACIÓN } \\
\text { DE PEARSON }\end{array}$} & $\begin{array}{l}\text { RSC } \\
\text { GOB }\end{array}$ & ROA & ROE & LIQ & END & TAM \\
\hline \multirow{3}{*}{$\begin{array}{l}\text { RSC } \\
\text { GOB }\end{array}$} & TOTAL & 1 & & & & & \\
\hline & En IBEX & 1 & & & & & \\
\hline & No IBEX & 1 & & & & & \\
\hline \multirow{3}{*}{ ROA } & TOTAL & 0,112 & 1 & & & & \\
\hline & En IBEX & 0,041 & 1 & & & & \\
\hline & No IBEX & 0,207 & 1 & & & & \\
\hline \multirow{3}{*}{ ROE } & TOTAL & $-0,103$ & 0,235 & 1 & & & \\
\hline & En IBEX & $-0,343$ & $-0,113$ & 1 & & & \\
\hline & No IBEX & 0,229 & $0,762 * *$ & 1 & & & \\
\hline \multirow{3}{*}{ LIQ } & TOTAL & $-0,359 * *$ & 0,089 & $-0,152$ & 1 & & \\
\hline & En IBEX & $-0,454^{*}$ & 0,152 & $-0,182$ & 1 & & \\
\hline & No IBEX & $-0,305$ & 0,012 & $-0,059$ & 1 & & \\
\hline \multirow{3}{*}{ END } & TOTAL & 0,169 & $-0,304 *$ & $0,369 * *$ & $-0,511^{* *}$ & 1 & \\
\hline & En IBEX & $-0,108$ & $-0,637 * *$ & $0,390 *$ & $-0,500 * *$ & 1 & \\
\hline & No IBEX & 0,420 & $-0,030$ & 0,356 & $-0,576^{* *}$ & 1 & \\
\hline \multirow{3}{*}{ TAM } & TOTAL & $0,544 * *$ & $-0,212$ & $-0,087$ & $-0,366^{* *}$ & $0,388^{* *}$ & 1 \\
\hline & En IBEX & $0,412 *$ & $-0,293$ & $-0,238$ & $-0,273$ & 0,161 & 1 \\
\hline & No IBEX & 0,366 & $-0,255$ & $-0,012$ & $-0,746^{* *}$ & $0,733^{* *}$ & 1 \\
\hline
\end{tabular}

*. La correlación es significante al nivel 0,05 (bilateral).

**. La correlación es significativa al nivel 0,01 (bilateral).

Fuente: Elaboración propia a partir de los datos obtenidos con IBM SPSS Statistics (versión 25.0). 
Por último, la cantidad de información vinculada al Gobierno Corporativo presenta una mayor similitud con la información medioambiental, ya que solo muestra relaciones significativas con respecto a la liquidez y al tamaño.

En el caso de la liquidez, la relación sigue siendo negativa aunque afecta a la muestra total de manera más significativa (al 1\%), que a la submuestra de empresas pertenecientes al IBEX (al 5\%).

Respecto al tamaño, coincide en la significatividad al $1 \%$ de la muestra total, aunque solo aparece relación significativa con la submuestra de empresas del IBEX con menor significatividad (5\%).

\section{Conclusiones}

En lo relativo al estudio de la relación entre la cantidad de información sobre RSC publicada y las variables analizadas se concluye:

- El tamaño es la única variable que aparece como significativa para el total de la muestra con respecto a los diferentes tipos de información. Esta circunstancia pone de manifiesto que las empresas más grandes tienen mayor propensión a publicar datos sobre los diferentes aspectos vinculados con la RSC, lo que puede derivarse de una búsqueda para justificar su comportamiento en este tipo de aspectos. Sin embargo, si se analizan las submuestras, el tamaño no afecta a la información publicada sobre aspectos sociales en el caso de las empresas incluidas en el IBEX, ni a la información sobre Gobierno Corporativo para las no incluidas en el citado índice.

- $\quad$ La Liquidez es la segunda variable que más vinculación muestra con la información publicada, aunque no aparece como significativa en el caso de la información Social. En todos los casos la relación es negativa lo que indica que las empresas más transparentes en información sobre RSC, tienen menos liquidez, lo que puede explicarse por los gastos derivados de obtener y publicar esta información. Por otra parte, hay diferencias entre los distintos subgrupos que muestran una relación significativa, ya que las empresas incluidas en el IBEX solo presentan la citada relación con respecto a la información de Gobierno Corporativo, pero no en los restantes aspectos.

La relación del Endeudamiento con la información publicada solo aparece como significativa para el caso de la información global, y únicamente para las empresas que no se encuentran incluidas en el IBEX. Por tanto, salvo en el caso mencionado, la transparencia en este tipo de información no supone un mayor o menor endeudamiento, lo que puede deberse a que la financiación del proceso de obtención y publicación de información se realiza tanto con recursos propios como ajenos.

Ni la Rentabilidad Económica ni la Financiera presentan relación significativa con la información publicada sobre RSC en ninguno de los aspectos analizados, lo que refleja que la transparencia en este tipo de información no afecta ni de forma positiva ni negativa a la rentabilidad obtenida por las empresas. Por tanto, la vinculación de las empresas de economía social y la RSC no puede considerarse como un elemento que impulse a este tipo de empresas. 
- $\quad$ Si se tienen los resultados del segundo subgrupo, como el más cercano a las empresas de economía social, se observa que el tamaño es un elemento determinante de la transparencia en materia de RSC, aunque no afectaría en materia de información sobre Gobierno Corporativo. La relación inversa entre liquidez y la transparencia se muestra en todos los aspectos analizados, mientras que también se aprecia un mayor endeudamiento en el caso de la transparencia en el índice global.

\section{Referencias bibliográficas}

Adams, C. A., Evans, R. (2004) Accountability, completeness, credibility and the audit expectations gap. Journal of Corporate Citizenship, 14, 97-115.

Ahmadi, A., Marfou, M., Sadidi, S. M. (2018) The Effect of Corporate Social Responsibility on Their Stock Return. Astra Salvensis, pp. 563-574.

Allouche, J. Laroche, P. (2005) A Meta-Analytical Investigation of the Relationship between Corporate Social and Financial Performance. Revue de Gestion des Ressources Humaines, $\mathrm{N}^{\circ}$ 57, pp. 18- 41.

Anderson, J.W. (1989) Corporate social responsibility: Guidelines for top management. ABC-CLIO. E.E.U.U.

Archel, P. (2003) La divulgación de la información social y medioambiental de la gran empresa española en el periodo 1994-1998: Situación actual y perspectivas, Revista Española de Financiación y Contabilidad, № 117, pp. 136-152.

Arimany, N., Puigvi, S., Sabatta, A. (2013) Análisis de los indicadores RSC/indicadores financieros en las empresas no financieras del IBEX 35. Revista Internacional de Género y Comercio, XI (21), pp. 1-9.

Aupperle, K.E., Carroll, A.B. y Hatfield, J.D. (1985) An Empirical Examination of the Relationship between Corporate Social Responsibility and Profitability. Academy of Management Journal, $\mathrm{N}^{\mathrm{0}} 28$, Vol. 2, pp. 446-463.

Barnea, A., Rubin, A. (2010) Corporate social responsibility as a conflict between shareholders. Journal of Business Ethics, $\mathrm{N}^{\mathrm{o}}$ 97, Vol. 1, pp. 71-86.

Barnett, M. (2007) Stakeholder influence capacity and the variability of financial returns to corporate social responsibility. Academy of Management Review, $\mathrm{N}^{\mathrm{o}}$ 32, Vol. 3, pp. 794-816.

Bebchuk, L. A., Weisbach, M. S. (2010) The state of corporate governance research. The review of financial studies, $\mathrm{N}^{\circ} 23$, Vol. 3, pp. 939-961.

Bonin, G. (2003) Die Leitung der Aktiengesellschaft zwischen Shareholder Value und Stakeholder-Interessen, Baden-Baden.

Campbell, D., Craven, B., Shrives, P. (2003) Voluntary social reporting in three FTSE sectors: a comment on perception and legitimacy. Accounting, Auditing \& Accountability Journal, № 16, Vol. 4, pp. 558-581.

Campbell, D. (2004) A longitudinal and cross-sectional analysis of environmental disclosure in UK companies- a research note. The British Accounting Review, No $^{\circ} 36$, Vol. 1, pp. 107-117.

Carrasco, I. (2007) Corporate Social responsibility, values and cooperation. International Advances in Economic Research, $\mathrm{N}^{\circ} 13$, Vol. 4, pp. 454-460.

Carroll, A. B. (1999) Corporate social responsibility: Evolution of a definitional construct. Business \& Society, № 38, Vol. 3, pp. 268-295. 
Castillo Clavero, A. (1985) La responsabilidad de la empresa en el contexto social: su articulación, gestión y control (Doctoral dissertation, Tesis Doctoral. Universidad de Málaga. Facultad Ciencias Económicas y Empresariales).

Castro Sanz, M. (2006) Las empresas de economía social y la responsabilidad social corporativa. Papeles de Economía Española, № 108, pp. 92-105.

Chang, Y., Chen, T. H., Shu, M. C. (2018) Corporate Social Responsibility, Corporate Performance, and Pay-Performance Sensitivity-Evidence from Shanghai Stock Exchange Social Responsibility Index. Emerging Markets Finance and Trade, $\mathrm{N}^{\circ}$ 54, Vol. 5, pp. 1183-1203.

Charlo Molina, M.J., Moya Clemente, I. y Muñoz Rubio, A.M. (2013) Factores diferenciadores de las empresas del índice de responsabilidad español. Cuadernos de Gestión, № 13, Vol. 2, pp. 15-37.

Charlo Molina, M.J. y Moya Clemente, I. (2010) El comportamiento financiero de las empresas socialmente responsables. Investigaciones Europeas de Dirección y Economía de la Empresa, $\mathrm{N}^{\circ}$ 16, Vol. 2, pp. 15-25.

Chivite Cebolla, M. y Enciso de Yzaguirre, V. (2015) Alcance de la creación de valor en las Empresas Responsables. CIRIEC-España, Revista de Economía Pública, Social y Cooperativa, $\mathrm{N}^{\circ} 85$.

Cho, C. H., Patten, D. M. (2007) The role of environmental disclosures as tools of legitimacy: A research note. Accounting, Organizations and Society, $\mathrm{N}^{\mathrm{o}} 32$, Vol. 7-8, pp. 639-647.

Collado, J.L. (2006) La Responsabilidad social corporativa y las cooperativas: aspectos y formulaciones comunes. En: XI Jornadas de Investigadores de Economía Social y Cooperativa. CIRIEC-España y CECOOP. Santiago de Compostela.

Comín, A. (2007) La responsabilidad social empresarial y la economía social. Cuadernos de Economía Social-CEPES, No 4, pp.1-6.

Cuevas Mejía, J. J., Maldonado García, S. y Escobar Váquiro, N. (2013) Aproximación a los factores que influyen en la divulgación de información sobre RSC en empresas de América Latina. Cuadernos de Contabilidad, № 14, Vol. 34, pp. 91-131.

De la Cuesta, M. y Valor, C. (2013) Evaluation of the environmental, social and governance information disclosed by Spanish listed companies. Social Responsibility Journal, $\mathrm{N}^{\circ}$ 9, Vol. 2, pp. 220-240.

De Villiers, C., Naiker, V. y Van Staden, C. J. (2011) The effect of board characteristics on firm environmental performance. Journal of Management, $\mathrm{N}^{\mathrm{o}} 37$, Vol. 6, pp. 16361663.

Dey, C. (2007) Social accounting at Traidcraft plc: a struggle for the meaning of fair trade. Accounting, Auditing \& Accountability Journal, No 20, Vol. 3, pp. 423-445.

Domench, P. A. (2003) La divulgación de la información social y medioambiental de la gran empresa española en el período 1994-1998: situación actual y perspectivas. Spanish Journal of Finance and Accounting/Revista Española de Financiación y Contabilidad, № 32, Vol. 117, pp. 571-601.

Elkington, J. (1998) Partnerships from cannibals with forks: The triple bottom line of 21 st-century business. Environmental Quality Management, № 8, Vol. 1, pp. 37-51.

España. Ley 11/2018, de 28 de diciembre, por la que se modifica el Código de Comercio, el texto refundido de la Ley de Sociedades de Capital aprobado por el Real Decreto Legislativo 1/2010, de 2 de julio, y la Ley 22/2015, de 20 de julio, de Auditoría de Cuentas, en materia de información no financiera y diversidad. Boletín Oficial del Estado, 29 de diciembre de 2018, núm. 314, 129833-129854. 
Escrig Olmedo, E., Muñoz Torres, M. J. y Fernandez Izquierdo, M. A. (2010). Socially responsible investing: sustainability indices, ESG rating and information provider agencies. International Journal of Sustainable Economy, № 2, Vol. 4, pp. 442-461.

Fernández, I. B., \& Arca, J. M. (2016). La economía social ante la actual crisis económica en la Comunidad Autónoma del País Vasco. REVESCO. Revista de Estudios Cooperativos, $\mathrm{N}^{\circ}$. 122, pp. 7-33.

Friedman, M. (1970) The Social Responsibility of Business is to Increase its Profits, The New Times Magazzine, $\mathrm{N}^{\circ}$ 13, pp. 32-33.

García Villalobos, J.C., Iturrioz del Campo, J., Mateu Gordon, J. L. y Palomo Zurdo, R. (2011) La percepción sobre la responsabilidad social en las sociedades cooperativas de trabajo asociado y las sociedades laborales: un análisis en el ámbito de la ciudad de Madrid. REVESCO. Revista de Estudios Cooperativos, Primer Cuatrimestre, № 104, pp. 102-124. DOI: 10.5209/rev_REVE.2011.v104.4.

Gray, R., Dey, C., Owen, D., Evans, R., Zadek, S. (1997) Struggling with the praxis of social accounting: stakeholders, accountability, audits and procedures. Accounting, Auditing \& Accountability Journal, No 10, Vol. 3, pp. 325-364.

Halbritter, G., Dorfleitner, G. (2015) The wages of social responsibility-where are they? A critical review of ESG investing. Review of Financial Economics, № 26, pp. 25-35.

Harjoto, M., Jo, H., Kim, Y. (2017) Is institutional ownership related to corporate social responsibility? The nonlinear relation and its implication for stock return volatility. Journal of Business Ethics, $\mathrm{N}^{\mathrm{o}}$ 146, Vol. 1, pp. 77-109.

Hoopes, D. G. \& Miller, D. (2006) Ownership preferences, competitive heterogeneity, and family-controlled businesses. Family Business Review, № 19, Vol. 2, pp. 89-101.

Jacobs, M., Karagozoglu, K. y Layish, D. (2012) Resolution of corporate financial distress: an empirical analysis of processes and outcomes. The Journal of Portfolio Management, $\mathrm{N}^{\mathrm{o}} 38$, Vol. 2, pp. 117-135.

Jensen, M.C., Meckling, W.H. (1976) Theory of the Firm: Managerial Behavior, Agency Costand Ownership Structure, Journal of Financial Economics, $\mathrm{N}^{\mathrm{o}} 3$, Vol. 4, pp. 305360.

Johnson-Young, E., Magee, R. G. (2019) The CSR paradox: when a social responsibility campaign can tarnish a brand. Corporate Communications: An International Journal.

Kim, S. (2019) The process model of corporate social responsibility (CSR) communication: CSR communication and its relationship with consumers' CSR knowledge, trust, and corporate reputation perception. Journal of Business Ethics, $\mathrm{N}^{\circ} 154$, Vol. 4, pp. 1143 1159.

Kurucz, E. C., Colbert, B. A., Wheeler, D. (2008) The business case for corporate social responsibility. In The Oxford handbook of corporate social responsibility, Oxford University Press, Oxford.

López González, E., Martínez Ferrero, J. y García Meca, E. (2019) Corporate social responsibility in family firms: A contingency approach. Journal of Cleaner Production, $\mathrm{N}^{\mathrm{o}} 211$, pp. 1044-1064.

Lorenzo, A. M. C., Serrán, M. C., \& Angulo, J. J. P. (2016). La economía social como alternativa de autoempleo. El perfil del emprendedor/a en las sociedades laborales andaluzas. REVESCO: Revista de estudios cooperativos, $\mathrm{N}^{\circ} .122$, pp. 59-85.

McWilliams, A., Siegel, D. (2000) Corporate social responsibility and financial performance: correlation or misspecification?. Strategic Management Journal, $\mathrm{N}^{\mathrm{o}} 21$, Vol. 5, pp. 603-609.

Madorran, C. y Garcia, T. (2016) Corporate social responsibility and financial performance: the Spanish case. Revista de Administración de Empresas, № 56, Vol. 1, pp. 20-28. 
Mahon, J.F. y Mcgovan, R.A. (1998) Modeling industry political dynamics. Business \& Society, No 37, Vol. 4, pp. 390-413.

McWilliams, A. y Siegel, D. (2001) Corporate Social Responsibility: A Theory of the Firm Perspective, Academy of Management Review, No 26, Vol. 1, pp. 117-127.

Medina Castaño, A. e Iturrioz del Campo, J. (2018) Análisis del impacto del valor añadido del gestor sobre el binomio Rentabilidad-Riesgo, medido mediante el ratio de Sharpe, en los Fondos de Inversión Socialmente Responsables. REVESCO. Revista de Estudios Cooperativos, Primer Cuatrimestre, No 127, pp. 181-203. DOI: 10.5209/REVE.59770.

Moskowitz, M. (1972) Choosing Socially Responsible Stocks, Business and Society Review, $\mathrm{N}^{\mathrm{o}} 1$, pp. 72-75.

Mozas Moral, A. y Puentes Poyatos, R. (2010) La responsabilidad social corporativa y su paralelismo con las sociedades cooperativas. REVESCO. Revista de Estudios Cooperativos, Tercer Cuatrimestre, № 103, pp. 75-100.

Nelling, E.; Webb, E. (2009) Corporate Social Responsability and Financial Performance: The virtuous circle revisited. Review of Quantitative Finance and Accounting, $\mathrm{N}^{\mathbf{0}} 32$, Vol. 2, pp. 197-209.

Nieto Antolín, M., Fernández Gago, R. y Cabeza García, L. C. (2012) La RSC en la relación entre composición del consejo y valor de mercado de la empresa. Papeles de Economía Española, $\mathrm{N}^{\circ} 132$, pp. 315-328.

Ndemanga, D. A., Koffi, E. T. (2009) Ownership structure, industry sector and corporate social responsibility (CSR) practices: the case of Swedish listed companies. Rapport nr.: Master Degree Project 2009.

Orlitzky, M.; Schmidt, F., y Rynes, S.L. (2003) Corporate Social and Financial Performance: A meta-Analysis. Organization Studies, No 24, Vol. 3, pp. 403-441.

Park, A., Ravenel, C. (2013) Integrating sustainability into capital markets: Bloomberg LP And ESG's quantitative legitimacy. Journal of Applied Corporate Finance, $\mathrm{N}^{\circ} 25$, Vol. 3, pp. 62-67.

Patten, D. M. (1992) Intra-industry environmental disclosures in response to the Alaskan oil spill: a note on legitimacy theory. Accounting, Organizations and Society, № 17, Vol. 5, pp. 471-475.

Pérez Almendros, C., Espinosa Anke, L. y Torres Valdés, R. (2013) Corporate social responsibility in the IBEX 35: a corpus-based study of CSR reports. Procedia-Social and Behavioral Sciences, $\mathrm{N}^{\circ}$ 95, pp. 612-620.

Puentes Poyatos, R. y Velasco Gámez., M.M. (2009) Importancia de las sociedades cooperativas como medio para contribuir al desarrollo económico, social y medioambiental, de forma sostenible y responsable. REVESCO. Revista de Estudios Cooperativos, № 99, pp. 104-119.

Sanz Santolaria, C. J. (2002) Guía de los principales ratios. Acciones e Investigaciones Sociales, $\mathrm{N}^{\mathrm{o}} 14$, pp. 137-152.

Socias Salvà, A. y Horrach Rosselló, P. (2013) Enfoque de la responsabilidad social y la transparencia en empresas de economía solidaria. CIRIEC-España, Revista de Economía Pública, Social y Cooperativa, No 77, pp. 267-297.

Steiner, G.A. (1971) Business and society. New York: Random House.

Sun, M., Nagata, K., Onoda, H. (2011) The investigation of the current status of socially responsible investment indices. Journal of Economics and International Finance, $\mathrm{N}^{\mathrm{o}} 3$, Vol. 13, pp. 676-684.

Testera Fuertes, A. y Cabeza García, L. (2013) Análisis de los factores determinantes de la transparencia en RSC en las empresas españolas cotizadas. Intangible Capital, $\mathrm{N}^{\circ}$ 9, Vol. 1, pp. 225-261. 
Ullman, A. (1985) Data in search of a theory: A critical examination of the relationship among social disclosure and economic performance measures. Academy of Management Review, No 19, Vol. 3, pp. 30-39.

Unión Europea. Directiva (UE) 2014/95/UE del Parlamento Europeo y del Consejo, de 22 de octubre de 2014, por la que se modifica la Directiva 2013/34/UE en lo que respecta a la divulgación de información no financiera e información sobre diversidad por parte de determinadas grandes empresas y determinados grupos. Diario Oficial de la Unión Europea, núm. 330, 15 de noviembre de 2014, 1-9.

Vázquez Oteo, O. (2015) Factores que influyen en la calidad y cantidad de Responsabilidad Social en las empresas españolas, Estudio de caso de las empresas del Ibex 35. CIRIECEspaña, Revista de Economía Pública, Social y Cooperativa, № 85, pp. 111-124.

Vázquez Oteo, O. V. y Cordero Sanz, C. (2007) Análisis empírico de la información emitida por las empresas del IBEX 35 en materia de responsabilidad social corporativa. Ekonomiaz: Revista Vasca de Economía, № 65, pp. 150-183.

Waddock, S. y Graves, S.B. (1997) The Corporate Social Performance- Financial performance link. Strategic Management Journal, No 18, Vol. 4, pp. 303-319. 